Christopher A. Hawkes BSC BMED SCI MD FRCPC

\title{
Factitious halothane detection during trig- ger-free anesthesia in a malignant hyperthermia susceptible patient
}

Purpose: To discuss the problems encountered when halothane was detected in a presumed 'clean' patient circuit during the 'trigger-free' anesthetic management of a known Malignant Hyperthermia Susceptible (MHS) patient for routine orthopedic surgery.

Clinical Features: A 29-yr-old MHS woman had a wrist arthroscopy/exploration/fusion under general anesthesia. During the course of the 'trigger-free' anesthetic the respiratory gas analyser detected end-tidal halothane in the patient circuit. The patient was disconnected from the circuit as attempts to identify the source of the readings were undertaken. After ruling out the presence of halothane by various clinical manoeuvres the patient was reconnected to the circuit without sequelae.

Conclusion: By exclusion the problem was presumed to be a factitious reading resulting from the respiratory gas analyser incorrectly identifying patient-expired methane as halothane.

Objectif : Faire le point sur les problèmes survenus lorsque de l'halothane a été détecté dans un circuit présumé «propre» pendant l'anesthésie, sans «agent déclenchant», d'une patiente susceptible d'hyperthermie maligne (SHM) au cours d'une intervention orthopédique de routine.

Éléments cliniques : Une femme de 29 ans SHM s'est présentée pour l'arthroscopie / exploration / fusion d'un poignet sous anesthésie générale. Pendant l'anesthésie sans «facteur déclenchant» d'hyperthermie, l'analyseur de gaz respiratoires a détecté la présence d'halothane dans le cirucuit en fin d'expiration. Le circuit a été débranché et on a tenté de vérifier la présence d'halothane. Mais cette possibilité a été exclue à la suite de diverses manoeuvres cliniques et on a rebranché le circuit, ce qui n'a laissé aucune séquelle à la patiente.

Conclusion : On a déduit que le problème provenait d'une fausse indication de l'analyseur de gaz respiratoires qui aurait incorrectement identifié le méthane expiré comme de l'halothane.

From the Department of Anesthesiology, Queen's University, Kingston General and Hotel Dieu Hospitals, Kingston, Ontario.

Address correspondence to: Dr. Christopher A. Hawkes, Department of Anesthesia, The Moncton Hospital, 135 MacBeath Avenue, Moncton, New Brunswick, ElC 6Z8. Phone: 506-857-5111; Fax: 506-857-5545; E-mail: chawk@nb.symparico.ca Accepted for publication March 14, 1999 
$\mathrm{N}$

EW pharmacological agents in the anesthesiologist's armamentarium have made providing anesthesia for Malignant Hyperthermia Susceptible (MHS) patients both safe and easy. Propofol infusions with opioid supplementation have replaced the triggering volatile agents, while the newer non-depolarizing muscle relaxants have allowed the avoidance of succinylcholine. The following report describes the problems encountered when the respiratory gas analyser falsely identified halothane in the breathing circuit of a known MHS patient during a 'non-triggering' general anesthetic.

\section{Case Report}

The patient was a 29 -yr-old, $55 \mathrm{~kg}$ woman with a sixmonth history of left wrist pain resulting from a hyperextension injury. A wrist arthroscopy/exploration and possible fusion with iliac crest bone graft was planned. The patient was fasting, with no reflux disease and had controlled asthma. Current medications included a fenoterol inhaler and ibuprofen. She was allergic to cefazolin. Past anesthetic history revealed a treated $\mathrm{MH}$ crisis six years previously and a subsequent positive caffeine-halothane contracture test. A recent wrist arthroscopy had been done with a brachial plexus block that the patient found very uncomfortable. She was reluctant to have a repeat regional anesthetic. Preoperative examination was unremarkable, with a normal airway and clear breath sounds bilaterally. She was agreeable to an intravenous (iv) block for the wrist arthroscopy/exploration, with the understanding that a general anesthetic would be required if an iliac crest bone graft was needed. The procedure was scheduled as the first case of the day.

In the operating room (OR), the $\mathrm{MH}$ protocol was in place. The Ohmeda Excel $210 \mathrm{SE}$ anesthesia machine had the vaporizers removed and had been flushed for $10 \mathrm{~min}$ with $\mathrm{O}_{2}\left(10 \mathrm{~L} \cdot \mathrm{min}^{-1}\right)$ as the ventilator cycled on an artificial lung. The $\mathrm{CO}_{2}$ absorber canisters had been removed, emptied, wiped out, and filled with new soda lime.

The patient was brought to the $\mathrm{OR}$, an intravenous catheter was inserted on the non-operative side and 1 $\mu \mathrm{g} \cdot \mathrm{kg}^{-1}$ fentanyl and $20 \mu \mathrm{g} \cdot \mathrm{kg}^{-1}$ midazolam were administered. An ip block was established with 3 $\mathrm{mg} \cdot \mathrm{kg}^{-1}$ lidocaine $0.6 \%$ using a double tourniquet inflated to $250 \mathrm{mmHg}$. More fentanyl $\left(1 \mu \mathrm{g} \cdot \mathrm{kg}^{-1}\right)$ was given due to discomfort from the block tourniquet as the lidocaine was injected. Within two minutes it became clear the patient would be unable to tolerate the tourniquet discomfort. General anesthesia was induced with $2.5 \mathrm{mg} \cdot \mathrm{kg}^{-1}$ propofol while the tourniquet remained inflated. A laryngeal mask airway
(LMA) was inserted and a propofol infusion was started $\left(180 \mu \mathrm{g} \cdot \mathrm{kg}^{-1} \cdot \mathrm{min}^{-1}\right)$ as $\mathrm{N}_{2} \mathrm{O} / \mathrm{O}_{2}\left(3 / 2 \mathrm{~L} \cdot \mathrm{min}^{-1}\right)$ were added. Spontaneous respiration resumed within five minutes with a HR of $60 \mathrm{bpm}, \mathrm{BP} 90 / 50 \mathrm{mmHg}$, $\mathrm{P}_{\mathrm{ET}} \mathrm{CO}_{2} 43 \mathrm{mmHg}$ and $\mathrm{SpO}_{2} 98 \%$. Meanwhile, the Datex AS/3 agent-specific respiratory gas analyser began to detect an expired end-tidal (ET) halothane (HAL) concentration of $0.3 \%$. The patient was immediately disconnected from the breathing circuit and the emergency ventilation equipment (Laerdal resuscitation self-inflating bag with $10 \mathrm{~L} \cdot \mathrm{min}^{-1} \mathrm{O}_{2}$ via wall source) was attached to the LMA. The anesthesia machine was again flushed with $20 \mathrm{~L} \cdot \mathrm{min}^{-1}$ air $/ \mathrm{O}_{2}$ into an artificial lung for five minutes. During this time the end-tidal halothane reading from the anesthesia circuit persisted, slowly decreasing from $0.3 \%$ to $0.1 \%$ as the patient's $\mathrm{HR}, \mathrm{BP}, \mathrm{P}_{\mathrm{ET}} \mathrm{CO}_{2}$ and $\mathrm{SpO}_{2}$ readings remained unchanged. The nasopharyngeal temperature was $36.2^{\circ} \mathrm{C}$. Meanwhile, the anesthesia aide consulted individuals in the Biomedical Engineering Department responsible for the maintenance of the anesthesia machines and monitors. A regional representative from Datex was also consulted.

The end-tidal halothane concentration on the monitor decreased to $0.1 \%$ and the monitor was turned off in attempts to reset the agent detection software. On restarting the monitor there was no detectable agent registering and the patient was reconnected to the circuit. The patient continued to breathe spontaneously (respiratory rates $<20 \mathrm{bpm}$ ) and other vital signs were stable. After five minutes back on the machine the circuit end-tidal halothane registered $0.3 \%$ again. The patient remained connected to the circuit for the remainder of the case that continued without incident for two hours. The LMA was removed with the patient awake and supine in the OR. Finally, a left stellate ganglion block was performed using $13 \mathrm{~mL}$ bupivacaine $0.25 \%$ as prophylaxis for the development of reflex sympathetic dystrophy in the injured limb.

The patient went to the Post Anesthesia Care Unit awake, alert and comfortable. Postoperatively, there were no problems and there was clinical evidence of a successful sympathetic block to the left arm. Following the standard four hours postoperative monitoring for MHS patients, she was admitted to the Overnight Stay Unit because of increased analgesia requirements. The patient was discharged home early the next morning in satisfactory condition.

\section{Discussion}

Intraoperative respiratory gas analysis has only recently come into widespread use and present guidelines to the practice of anesthesia encourage ${ }^{1}$ the use of such mon- 
itors. The respiratory gases of most interest to anesthesiologists include $\mathrm{O}_{2}, \mathrm{CO}_{2}, \mathrm{~N}_{2} \mathrm{O}$, and the potent inhaled anesthetic agents. Depending on the gas to be measured, there are a number of technologies available to make the analysis and present the data. The applications and limitations of each technology are described in standard anesthesia equipment textbooks. ${ }^{2}$

The issue in this case was whether halothane was present in the anesthesia circuit, with the potential triggering of a $\mathrm{MH}$ crisis. After discussions with the Biomedical Engineering Department and a Datex representative it was felt there were two likely explanations. First, the gas analyser might have been detecting residual halothane in the anesthesia machine as it leached out from rubber gaskets and other soluble sites. The alternative was that the patient had some undetermined breath-expired substance that the gas analyser incorrectly identified as halothane.

A number of factors lead to the belief that the machine in use was vapor-free despite the described readings. No agent was detected during the preoperative cycling of the ventilator implying the substance was not coming from the anesthesia machine. Doyle describes an incident in which a factitious halothane reading was the result of an anomalous vapor present in the anesthesia machine. ${ }^{3} \mathrm{He}$ found the isopropyl alcohol $70 \%$ used to clean their anesthetic circuit components gave an inspired/expired reading of halothane $6 \%$. A check of the patient circuit with an artificial lung using the respiratory gas analyser could detect such problems preoperatively. In fact, newer agent analysers will identify alcohol in the patient circuit that may originate either from a disinfectant containing ethanol or propane or from the patient's breath. ${ }^{4}$ In this case, the agent detected was only an expired reading, implying a patient source. While the use of a circle system at semiclosed fresh gas flows might allow the gas eventually to be detected as 'inspired', it did not during the two hour case. In addition, sevoflurane and isoflurane vaporizers were removed preoperatively, and it had been some days and numerous anesthetics since a halothane vaporizer had been on the machine. This made the presence of halothane even more unlikely. The strongest evidence that it was patient generated was the placement of the respiratory gas analyser between the LMA and the Laerdal bag which continued to show expired 'halothane'. The patient was therefore reconnected to the anesthesia machine and circuit confident that it was a 'clean machine' and safe to continue the procedure.

Postoperative questioning of the patient regarding ingestion of alcohol or other unusual substances over the past 24-hr period was negative. Presenting this case to the GASNet Anesthesiology Discussion
Group ${ }^{5}$ was more helpful. It was suggested that the halothane detected might be the result of patientexpired methane. Indeed, there is a considerable amount of literature regarding the phenomenon of methane production in humans over the past two decades. Peled $e t a l$. studied factors affecting methane formation (a result of the action of colonic flora) and found that $50 \%$ of their control patients produced breath methane. ${ }^{6}$ There have been reports in the anesthesia literature of patient-expired methane as well. Versichelen et al. found two-thirds of 26 patients analyzed during gynecological surgery had false halothane readings attributed to breath methane. ${ }^{7}$ Using closed-system anesthesia during total intravenous anesthesia they found the agent analyser (using an infrared spectrometer, measuring a wavelength of $3.3 \mu \mathrm{m}$ ) falsely indicated halothane readings in 18 patients. The measured 'halothane' concentrations progressively increased in the closed system during the 60-min study period and correlated with the accumulation of methane (measured by a separate photoacoustic infrared method at a wavelength of $3.6 \mu \mathrm{m}$ ). The Datex AS $/ 3$ respiratory gas analyser also uses an infrared light source to measure the inhalational agent absorption peak in the $3.3 \mu \mathrm{m}$ range, ${ }^{8}$ and a similar problem may have occurred in this case.

Locally, other department members have noticed potential false halothane readings while using the Datex AS/3 system. This system automatically identifies the agent in use and can detect instances when two different agents are present in the patient circuit. Such dual readings typically arise when, for economic reasons, an inhalational induction is performed with one agent and maintenance of the anesthetic is with another. There have been times when both halothane and another agent have been measured, even though halothane was not in use. This could potentially represent patientexpired methane. Review of the Datex AS $/ 3$ User's Reference Manual warns that some hydrocarbons (acetone and methane) may cause an 'Agent mixture' message to appear. ${ }^{4}$ Based on the discussion above, and this may occur more often than we think.

Future studies might focus on the clinical correlation between the methane concentration and the endtidal halothane readings, and whether these might be additive. As Versichelen et al. point out, false halothane readings may incite the lowering of vaporizer output, with the inherent danger of light anesthesia and awareness. During closed-system anesthesia in particular, they recommend the use of techniques where agent analysis is not influenced by the presence of methane (higher infrared wavelengths of 10-13 $\mu \mathrm{m}$; mass spectrometry; Raman scattering). 
In summary, this report describes the problems encountered when halothane was detected in a presumed 'clean' patient circuit during the anesthetic management of a patient known to be $\mathrm{MH}$ susceptible. The problem was felt to be the result of patientexpired methane incorrectly detected by the respiratory gas analyser as halothane. The anesthesiologist chose to continue to use the unchanged breathing circuit without incident and the postoperative course was uncomplicated.

\section{References}

1 CAS Guidelines to the Practice of Anaesthesia. Can J Anaesth 1997; 44(Suppl): 11.

2 Eisenkraft JB, Raemer DB. Monitoring gases in the anesthesia delivery system. In: Ehrenwerth J, Eisenkraft JB (Eds.). Anesthesia Equipment. Principles and Applications, Ist ed. St. Louis: Mosby-Year Book Inc., 201-20.

3 Doyle DJ. Factitious readings from anaesthetic agent monitors (Letter). Can J Anaesth 1988; 35: 667.

4 Datex-Engstrom AS/3 Anaesthesia Monitor User's Reference Manual. July 1997. Finland: Datex-

Engstrom Division, Instrumentarium Corp., Manual No. 892946-0, 20-9.

5 Ruskin KJ, Kofke WA, Turndorf H. The Anesthesiology Discussion Group: development of a new method of communication between anesthesiologists. Anesth Analg 1995; 81: 163-6.

6 Peled $\Upsilon$, Weinberg $D$, Hallak A, Gilat $T$. Factors affecting methane production in humans. Gastrointestinal diseases and alterations of colonic flora. Dig Dis Sci 1987; 32: 267-71.

7 Versichelen L, Rolly G, Vermeulen H. Accumulation of foreign gases during closed-system anaesthesia. $\mathrm{Br} \mathrm{J}$ Anaesth 1996; 76: 668-72.

8 Datex AS/3TM Anaesthesia Monitor Service Manual. September 1993. Helsinki Finland: Datex/Division of Instrumentarium Corp., Manual No. 882580, 3-1. 